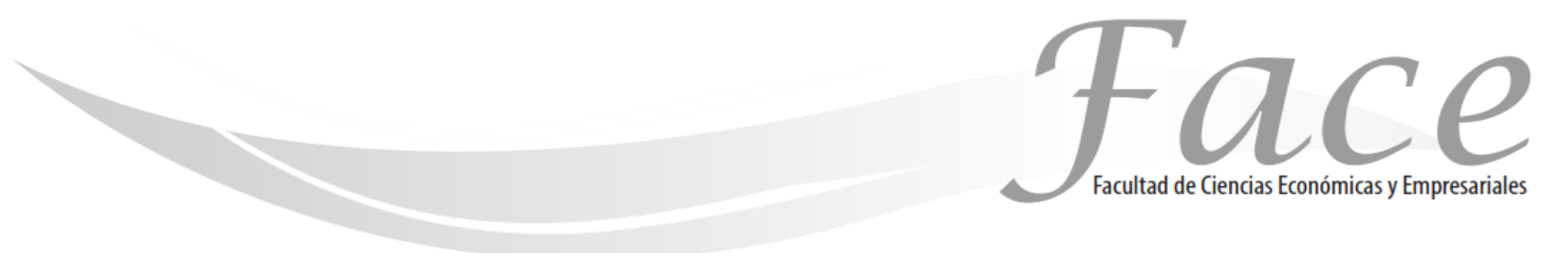

ISSN Impreso: 1794-9920

ISSN Electrónico: 2500-9338

Volumen 19-N¹

Año 2019

Págs. $50-57$

(c) (i) $\odot$

\title{
DISTANCIA DE HAMMING COMO INSTRUMENTO PARA MEDIR LA CERCANÍA A UN PRESIDENTE IDEAL: ESTUDIO APLICADO EN ANDRÉS MANUEL LÓPEZ OBRADOR
}

\author{
Mauricio Ortigosa Hernández * \\ ORCID: https://orcid.org/0000-0003-1461-4962
}

Fecha de Recepción: Febrero 27 de 2019

Fecha de Aprobación: Mayo 14 de 2019

\section{Resumen:}

Las elecciones presidenciales del $1^{\circ}$ de Julio del 2018 en México, resultaron sin precedentes en la historia de la democracia en México, ya que fue contundente la victoria de Andrés Manuel López Obrador (AMLO) por parte del partido Movimiento de Regeneración Nacional (MORENA). A partir de esos comicios, surgió la siguiente pregunta: ¿acaso los seguidores y simpatizantes de MORENA perciben a López Obrador como el presidente ideal que debería tener México? Para dar respuesta a esta pregunta, el presente estudio construye la imagen de un presidente ideal y la percepción del ganador para los diferentes partidos políticos. Posteriormente, utilizando la distancia de Hamming, se obtienen mediciones que permiten establecer el acercamiento (o alejamiento) entre la imagen de un presidente ideal y la percepción que tienen de López Obrador en cada uno de los partidos políticos. El resultado de la investigación muestra que los simpatizantes de MORENA, perciben a López Obrador como el presidente más cercano al presidente ideal para México.

Palabras clave: marketing político, presidente ideal, distancia de Hamming, subconjunto borroso.

\footnotetext{
* Doctorado en Estudios Empresariales por la Universidad de Barcelona, España. Master en Marketing en la Universidad Autónoma de Madrid. Maestría en Administración de Empresas en el Instituto Tecnológico y de Estudios Superiores de Monterrey (ITESM) en la ciudad de México. Docente de la Facultad de Economía y Negocios en la Universidad Anáhuac México Contacto: mauricio.ortigosa@anahuac.mx
} 


\title{
DISTANCE OF HAMMING AS AN INSTRUMENT TO MEASURE THE CLOSENESS TO AN IDEAL PRESIDENT: STUDY APPLIED IN ANDRÉS MANUEL LÓPEZ OBRADOR.
}

\begin{abstract}
:
The presidential elections of July 12018 , they were unprecedented in the history of democracy in Mexico, since the victory of Andrés Manuel López Obrador (AMLO) was overwhelming, by the political party of Movimiento de Regeneración Nacional (MORENA). From the elections the next question arose: ¿Does the followers and supporters of MORENA perceive López Obrador as the ideal president for Mexico? In order to provide an answer, the present study builds the image of an ideal president and the perception that the other political parties had of the winner. Later, using the distance of Hamming different measurements are obtained that allow to establish the approach (or distance) between the image of the ideal president and the perception of López Obrador among the different political parties. The result of the research shows that the supporters of MORENA perceive López Obrador as the president closest to the image of the ideal president for Mexico.
\end{abstract}

Keywords: Political Marketing, Ideal President, Hamming Distance, Fuzzy Subsets.

\section{DISTÂNCIA DO ENCONTRO COMO INSTRUMENTO DE MEDIÇÃO DA CLOSEENDE A UM PRESIDENTE IDEAL: ESTUDO APLICADO EM ANDRÉS MANUEL LÓPEZ OBRADOR.}

\section{Resumo:}

As eleições presidenciais de 1 de julho de 2018 foram inéditas na história da democracia no México, uma vez que a vitória de Andrés Manuel López Obrador (AMLO) foi esmagadora, pelo partido político do Movimento de Regeneração Nacional (MORENA). Nas eleições, surgiu a seguinte pergunta: "Os seguidores e apoiadores da MORENA percebem López Obrador como o presidente ideal para o México? A fim de fornecer uma resposta, o presente estudo constrói a imagem de um presidente ideal e a percepção que os outros partidos políticos tiveram do vencedor. Posteriormente, utilizando a distância de Hamming se conseguem diferentes medidas que permitem estabelecer a aproximação (ou distância) entre a imagem do presidente ideal e a percepção de López Obrador entre os diferentes partidos políticos. 0 resultado da pesquisa mostra que os partidários da MORENA percebem López Obrador como o presidente mais próximo da imagem do presidente ideal para o México.

Palavras-chave: Marketing Político, Presidente Ideal, Distância de Hamming, Subconjuntos Fuzzy. 
ISSN: 1794-9920 Impreso / Electrónico 2500-9338

Enero - Julio de 2019 - Volumen 19 Número 2, Año 2019 Págs. 50 - 57

\section{INTRODUCCIÓN:}

Desde hace varias décadas, diversas investigaciones, indican que las personas construyen imágenes mentales de lo que consideran que debería ser un presidente ideal. Después, tienden a comparar a los candidatos reales que compiten para la presidencia con esas imágenes ideales (García, D’Adamo y Zubieta, 2016). Cuando un político puede avalar a través de su propia biografía y cualidades personales un proyecto de gobierno, brinda una garantía para mejorar no sólo el dialogo político, sino la misma actuación política (Orejuela, 2009). El mismo autor menciona que hay tres dimensiones que configuran la imagen de un político: lo que el candidato es, lo que el candidato proyecta y lo que perciben los votantes. En el presente trabajo nos referimos a la imagen que perciben los simpatizantes. Lo anterior, hace que la imagen percibida sea una construcción mental de carácter subjetiva, lo que da pauta a utilizar la noción de subconjunto borroso.

La aportación del presente trabajo al marketing político, es la utilización de instrumentos novedosos para construir la imagen de políticos utilizando el concepto de subconjunto borroso.

Con dicho concepto, tratamos de dar respuesta a la pregunta: ¿acaso los seguidores y simpatizantes de MORENA perciben a López Obrador como el presidente ideal que debería tener México?

La pregunta anterior, da lugar a establecer la siguiente hipótesis:

"Los simpatizantes del partido MORENA perciben a Andrés Manuel López Obrador como la figura más cercana al perfil de un presidente ideal, comparado con la percepción que tienen los simpatizantes de cualquier otro partido político".

Para lograr lo anterior, adaptamos un modelo de Gestión de Recursos Humanos propuesto por Gil Aluja (2002) en el marco de la Teoría de los subconjuntos borrosos. En nuestro caso, establecemos el perfil ideal para el puesto de presidente de la República Mexicana. Posteriormente, construimos para cada partido político, la percepción de López Obrador donde finalmente, con el uso de la distancia de Hamming, encontramos la cercanía (o alejamiento) entre el perfil ideal de presidente y la percepción de López Obrador para cada partido político.

\section{MARCO TEÓRICO. Elementos previos al desarrollo de la distancia de Hamming:}

En la historia política contemporánea de México, se han realizado estudios sobre la percepción de líderes en épocas de elecciones. Ejemplo de esto, es el desarrollado por Merolla y Zechmeister (2009) en las elecciones mexicanas presidenciales del 2006. Dicho estudio mostró que Felipe Calderón y López Obrador, candidatos punteros a la presidencia, a pesar de tener percepciones similares en los votantes en cuanto a su fortaleza y carisma, ambos candidatos promovían imágenes muy diferentes. Felipe Calderón como un buen administrador y orientado a la clase media y alta. López Obrador, como un populista y viendo el interés de los pobres ofreciendo propuestas ambiciosas. El estudio mostró evidencia que las condiciones contextuales (amenazas, riesgos, etc.) tienen un efecto importante en la forma en que el electorado percibe a los líderes. Deusdad (2003), menciona que el carisma es una característica intrínseca de las sociedades e inseparable del liderazgo político.

A lo largo de varias décadas, se han realizado un gran número de investigaciones sobre imagen de figuras políticas o candidatos a puestos públicos (Zamora y Losada, 2011; Zakrizevska, 2012; Diemidova, Adamovska y Garasiuk, 2018; Roets y Van Hiel, 2009; Ogneva y Kalmykov, 2017; Orejuela, 2009). Incluso, hay autores que se han especializado en estudiar la imagen ideal de candidatos presidenciales, tema que es de interés en el presente documento.

A manera de síntesis, Hudy y Capelos (2002) citado por García, D’Adamo y Zubieta (2016), mencionan que las investigaciones en cuestión de imagen de candidatos y líderes presidenciales coinciden en indicar que hay ciertas cualidades ideales que no se han modificado con el transcurso del tiempo, ejemplo de ellas son liderazgo, empatía, competencia, entre otros atributos.

En esta investigación, al estudiar estas cualidades a través del uso de los subconjuntos borrosos, es necesario comprender su significado descrito de forma breve en las siguientes líneas. 
Mauricio Ortigosa Hernández

Noción de subconjunto borroso.

Gil Aluja (2002) menciona que la noción de conjunto, constituye una base sólida sobre la cuál es posible edificar construcciones formales de capital importancia. El significado intuitivo de la palabra conjunto es simple; se trata de una colección de objetos, físicos o mentales perfectamente bien especificados. Tomando como "referencia" un conjunto muy amplio, se puede considerar casi una infinidad de subconjuntos. Al conjunto de referencia se le acostumbra denominar "conjunto referencial".

En nuestro estudio, vamos a proponer un conjunto referencial como una colección de cualidades, características 0 singularidades que describen las características del perfil ideal al puesto de trabajo, en este caso, para presidente de la república $\left(\mathrm{C}_{1}, \mathrm{C}_{2}, . ., \mathrm{C}_{\mathrm{n}}\right)$.

Bajo la óptica tradicional, resulta cómodo representar al conjunto referencial asignando el valor de 1 si cumple el máximo nivel requerido en cada cualidad, característica 0 singularidad al puesto de trabajo, y con valor de cero, representando el nivel mínimo requerido en cada atributo.

Por ejemplo, si $\mathrm{P}_{\mathrm{I}}$ es el perfil o descriptor al puesto de trabajo, se puede representar de la forma siguiente:

$\mathrm{P}_{\mathrm{l}}=$\begin{tabular}{|c|c|c|c|c|c|}
\hline $\mathrm{C}_{1}$ & $\mathrm{C}_{2}$ & $\ldots$ & $\mathrm{C}_{\mathrm{i}}$ & $\ldots$ & $\mathrm{C}_{\mathrm{n}}$ \\
\hline 1 & 0 & & 1 & & 0 \\
\hline
\end{tabular}

No obstante, si matizamos dicha presencia o ausencia requerida en cada atributo, esto constituye como lo menciona Gil Aluja (2002), la idea básica de la teoría de los subconjuntos borrosos, permitiendo asignar un valor comprendido entre 1 y 0 , incluyendo los valores extremos. Las cifras colocadas en cada celda son llamados por los matemáticos "valores de la función característica de pertenencia" y se acostumbra a representar dichas cifras con la letra griega $\mu$. Por ejemplo:

$\mathrm{P}_{\mathrm{l}}=$\begin{tabular}{|l|l|l|l|l|l|}
\hline $\mathrm{C}_{1}$ & $\mathrm{C}_{2}$ & $\ldots$ & $\mathrm{C}_{\mathrm{i}}$ & $\ldots$ & $\mathrm{C}_{\mathrm{n}}$ \\
\hline$\mu_{1}$ & $\mu_{2}$ & & $\mu_{\mathrm{i}}$ & & $\mu_{\mathrm{n}}$ \\
\hline
\end{tabular}

Así, la noción de subconjunto borroso resulta idónea para este trabajo. Estos elementos previos que hemos puesto en estas líneas, permiten en el estudio empírico, seleccionar un conjunto de cualidades, características 0 singularidades para que el ciudadano se forme una imagen mental acerca de cómo debería de ser un presidente ideal, posteriormente con ese mismo conjunto de cualidades, características o singularidades se construye la percepción que tienen de López Obrador para cada partido político. Con esta información, usamos la distancia de Hamming para determinar el acercamiento (o alejamiento) entre estos dos subconjuntos borrosos. Pero antes describimos la propuesta metodológica para aplicarla en el estudio empírico.

\section{METODOLOGIA:}

\subsection{Propuesta Metodológica}

Gil Aluja (2002) menciona que una solución para determinar un candidato para ocupar un puesto de trabajo, se puede resolver trabajando las siguientes etapas, las cuales las adaptamos a nuestro estudio donde el puesto de trabajo es la Presidencia de la República Mexicana.

Etapa 1. Establecer una lista de atributos. Se traduce en determinar y enumerar las cualidades, características y singularidades más significativas que debe tener el candidato para realizar una buena labor en el puesto de trabajo (presidente de la república en nuestro caso).

Etapa 2. Establecer el nivel ideal requerido de cada atributo. Esto se realiza fijando para cada cualidad, característica o singularidad, el nivel que debería poseer el candidato perfecto. En nuestro caso, es equivalente a encontrar la imagen del "presidente ideal".

Dicha imagen resulta idónea expresarla a través de un subconjunto borroso que actúa como descriptor al puesto de trabajo, estableciendo valoraciones objetivas y/o estimaciones numéricas subjetivas. Por tanto, se puede expresar como:

$P_{l}=$\begin{tabular}{|l|l|l|l|l|l|}
\hline$C_{1}$ & $C_{2}$ & $\ldots$ & $C_{i}$ & $\ldots$ & $C_{n}$ \\
\hline$\mu_{1}$ & $\mu_{2}$ & & $\mu_{i}$ & & $\mu_{n}$ \\
\hline
\end{tabular}

Donde $\mathrm{C}_{\mathrm{i}}$ representa las cualidades, características 0 singularidades para $i=1,2, \ldots, n$ y 
ISSN: 1794-9920 Impreso / Electrónico 2500-9338

Enero - Julio de 2019 - Volumen 19 Número 2, Año 2019 Págs. 50 - 57

$\mu_{\mathrm{i}}=$ representa el nivel ideal requerido para cada atributo desde $i=1,2, \ldots, n$ y son llamados "valores de la función característica de pertenencia" como se mencionó en el apartado anterior.

Etapa 3. Percepción del actual presidente en el puesto. Vamos a realizar una adaptación a lo propuesto por Gil Aluja (2002). En nuestro estudio, ya que el puesto de trabajo ya está ocupado y estará durante el período del 2018-2024, entonces determinamos para cada partido político, sólo la percepción de López Obrador utilizando los atributos de la etapa 1.

Supongamos que dichas valuaciones son expresadas a través de los siguientes subconjuntos borrosos (uno por cada partido político):

$$
\begin{aligned}
& P_{1}=\begin{array}{|c|c|c|c|c|c|}
\hline C_{1} & C_{2} & C_{3} & C_{4} & \ldots & C_{n} \\
\hline \mu_{11} & \mu_{21} & \boldsymbol{\mu}_{31} & \boldsymbol{\mu}_{41} & \ldots & \mu_{n 1} \\
\hline
\end{array} \\
& P_{2}=\begin{array}{|c|c|c|c|c|c|}
\hline C_{1} & C_{2} & C_{3} & C_{4} & \ldots & C_{n} \\
\hline \mu_{12} & \mu_{22} & \boldsymbol{\mu}_{32} & \boldsymbol{\mu}_{42} & \ldots & \mu_{n 2} \\
\hline
\end{array} \\
& P_{3}=\begin{array}{|c|c|c|c|c|c|}
\hline C_{1} & C_{2} & C_{3} & C_{4} & \ldots & C_{n} \\
\hline \mu_{13} & \mu_{23} & \boldsymbol{\mu}_{33} & \boldsymbol{\mu}_{43} & \ldots & \mu_{n 3} \\
\hline
\end{array} \\
& P_{4}=\begin{array}{|c|c|c|c|c|c|}
\hline C_{1} & C_{2} & C_{3} & C_{4} & \ldots & C_{n} \\
\hline \mu_{14} & \boldsymbol{\mu}_{24} & \boldsymbol{\mu}_{34} & \boldsymbol{\mu}_{44} & \ldots & \boldsymbol{\mu}_{n 4} \\
\hline
\end{array}
\end{aligned}
$$

Etapa 4. Distancia de Hamming. Una vez conocidas las anteriores valuaciones, iniciamos la última fase del proceso, cuyo carácter es básicamente técnico y se resuelva con ayuda de cualquier hoja de cálculo. Se trata de establecer una cercanía (o alejamiento) con la distancia de Hamming para los subconjuntos borrosos mostrados anteriormente:

$$
P_{1} \leftrightarrow P_{1} \quad, \quad P_{1} \leftrightarrow P_{2} \quad, \quad P_{1} \leftrightarrow P_{3} \quad y \quad P_{1} \leftrightarrow P_{4}
$$

En nuestro estudio, la distancia refleja el acercamiento entre la imagen del presidente ideal y la percepción de López Obrador para cada partido político.

Como resultado adicional, se puede obtener un orden de acercamiento (o alejamiento) entre los 4 partidos políticos.

Lo anterior nos permite comprobar la hipótesis que afirma que los simpatizantes del partido MORENA, que gobierna el país en el período 2018-2014, perciben a López Obrador como el presidente más cercano al perfil de un presidente ideal comparado con la percepción de López Obrador de cualquier otro partido político.

Por su sencillez y operatividad, Kaufmann y Gil Aluja (1992) definen la distancia de Hamming explícitamente para dos subconjuntos borrosos como la diferencia en valor absoluto, de los valores de la función característica de pertenencia para cada elemento del conjunto referencial:

$$
\mathrm{D}(\mathrm{A}, \mathrm{B})=\sum_{i=1}^{n}\left|\mu_{\mathrm{A}}\left(\mathrm{x}_{\mathrm{i}}\right)-\mu_{\mathrm{B}}\left(\mathrm{x}_{\mathrm{i}}\right)\right|
$$

Los mismos autores recomiendan utilizar la distancia relativa de Hamming $\delta$, sobre todo cuando se realizan comparaciones entre diferentes subconjuntos borrosos (como es nuestro caso). Para su obtención basta con dividir la distancia absoluta obtenida entre el número de elementos considerados. De esta forma la distancia pertenece al intervalo $[0,1]$ donde entre más próximo a cero, más cerca están los subconjuntos que se comparan.

$$
\delta(A, B)=\frac{1}{n} D(A, B)
$$

Para nuestro estudio, la distancia de Hamming tiene un inconveniente: se parte de la premisa que el nivel de percepción en cada cualidad, característica o singularidad respecto al nivel ideal de cada elemento del subconjunto borroso (descriptivo), resulta tan malo no llegar como sobrepasar el nivel ideal; esto se ve reflejado al tomar el valor absoluto de cada diferencia. Por ejemplo, si la cualidad, característica o singularidad es el "Carisma", consideramos que tan malo es estar por debajo del nivel ideal (genera imagen desagradable) como por arriba de dicho nivel (puede ganar simpatizantes de corta duración).

\subsection{Aplicación de la Metodología: Estudio en Andrés Manuel López Obrador}

Con el propósito de aplicar la metodología descrita en el apartado anterior, se obtuvo una muestra de simpatizantes de los 4 partidos políticos más importantes que participaron en la contienda. No se tomaron en cuenta los partidos pequeños que hicieron coalición, excepto el PRD que se alió con el PAN. La muestra total fue de 320 entrevistas distribuidos en los 4 partidos políticos: 80 del Partido de Movimiento de Regeneración Nacional (MORENA), 80 del Partido Acción Nacional (PAN); 80 del Partido Revolucionario Institucional (PRI) y finalmente 80 del Partido de la Revolución Democrática (PRD). 
Mauricio Ortigosa Hernández

Etapa 1. Establecer una lista de atributos. Se ha seleccionado una lista de 10 atributos. Cabe aclarar que no se pretende que sea una lista exhaustiva, pero consideramos que es un número aceptable para poder describir las principales cualidades, características 0 singularidades necesarias para un puesto presidencial. Dichos atributos se han utilizado en diferentes trabajos realizados por autores tales como: Ogneva y Kalmykov, 2017; Orejuela, 2009; Zamora y Losada, 2011; Kinder, Peters, Abelson y Fiske, 1980; Zakrizevska, 2012; García, D’Adamo y Zubieta, 2016; Berrocal, 2004; Martín, 2002; D'Adamo, García y Pastore, 2015; entre otros autores.

Los atributos seleccionados se muestran en el Cuadro 1.

\begin{tabular}{|l|c|}
\hline \multicolumn{2}{|c|}{$\begin{array}{c}\text { Cuadro 1. Cualidades, características o } \\
\text { singularidades seleccionadas para el perfil } \\
\text { ideal de presidente de la República } \\
\text { Mexicana }\end{array}$} \\
\hline $\mathrm{C}_{1}$ & Capacidad de tomar decisiones \\
\hline $\mathrm{C}_{2}$ & Honestidad \\
\hline $\mathrm{C}_{3}$ & Experiencia política \\
\hline $\mathrm{C}_{4}$ & Credibilidad \\
\hline $\mathrm{C}_{5}$ & Carisma \\
\hline $\mathrm{C}_{6}$ & Liderazgo \\
\hline $\mathrm{C}_{7}$ & Apariencia física \\
\hline $\mathrm{C}_{8}$ & Oratoria \\
\hline $\mathrm{C}_{9}$ & Nacionalismo \\
\hline $\mathrm{C}_{10}$ & Empatía \\
\hline
\end{tabular}

Etapa 2. Establecer el nivel ideal requerido de cada atributo. Para obtener el perfil ideal, se realizaron 320 entrevistas en la zona metropolitana de la ciudad de México y municipios adyacentes tales como Huixquilucan, Naucalpan de Juárez, etcétera. obteniendo el siguiente subconjunto borroso que representa la descripción del Presidente ideal de dichas entrevistas. Los resultados, se obtuvieron como un promedio de toda la muestra con un decimal y se expresa a continuación, bajo la forma de subconjunto borroso:

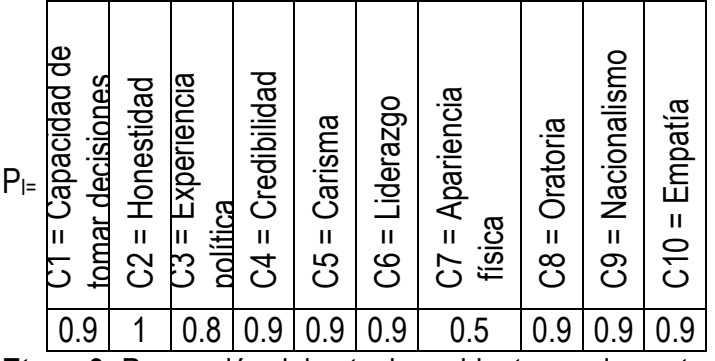

Etapa 3. Percepción del actual presidente en el puesto. Ya que el puesto de trabajo ya está ocupado por el período 2018-2024, sólo obtenemos la percepción que tienen los simpatizantes de los 4 partidos más importantes de López Obrador. Esto lo realizamos, construyendo 4 subconjuntos borrosos con los mismos atributos de la etapa 2.

Percepción de Andrés Manuel López Obrador en el PRI:

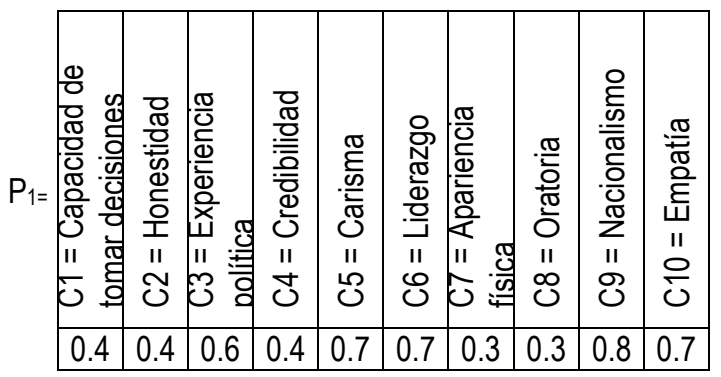

Percepción de Andrés Manuel López Obrador en el PAN:

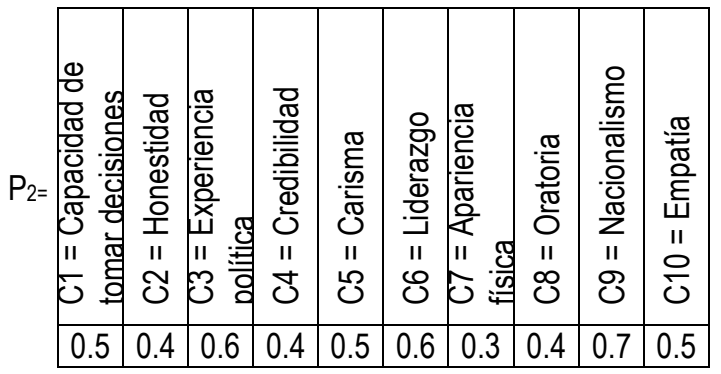

Percepción de Andrés Manuel López Obrador en el PRD:

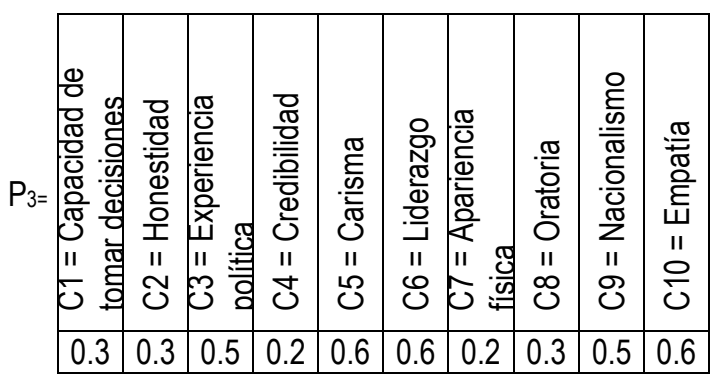


ISSN: 1794-9920 Impreso / Electrónico 2500-9338

Enero - Julio de 2019 - Volumen 19 Número 2, Año 2019 Págs. 50 - 57

Percepción de Andrés Manuel López Obrador en MORENA:

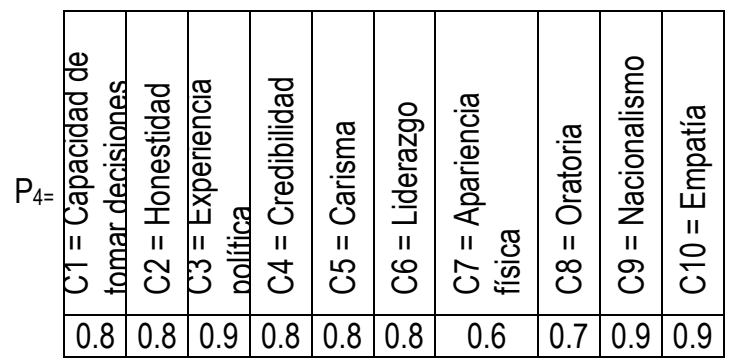

Etapa 4. Distancia de Hamming. Finalmente, vamos a utilizar la distancia de Hamming, para obtener numéricamente el acercamiento (o alejamiento) de la imagen del presidente ideal $\left(P_{I}\right)$ y la percepción de López Obrador para cada partido político $\left(P_{i}\right) i=1,2,3$ y 4 :

$$
P_{1} \leftrightarrow P_{1}, \quad P_{1} \leftrightarrow P_{2} \quad, \quad P_{1} \leftrightarrow P_{3} \quad \text { y } \quad P_{1} \leftrightarrow P_{4}
$$

Cabe recordar que al aplicar la distancia de Hamming, se parte de la premisa es que es tan malo no llegar al nivel exigido como sobre pasarlo. Sin embargo, por su sencillez optamos utilizar este criterio de distancia. Adaptando la notación a nuestro problema, calculamos la distancia de Hamming para cada partido político $\left(\mathrm{P}_{\mathrm{i}}\right)$ con respecto a la imagen del presidente ideal $\left(\mathrm{P}_{\mathrm{I}}\right)$ :

$$
\begin{gathered}
\mathrm{D}\left(\mathrm{P}_{\mathrm{I}}, \mathrm{P}_{\mathrm{i}}\right)=\sum_{i=1}^{n}\left|\mu_{\mathrm{P}}\left(\mathrm{x}_{\mathrm{i}}\right)-\mu_{\mathrm{P}}\left(\mathrm{x}_{\mathrm{i}}\right)\right| \text { distancia de Hamming } \\
\text { para cada } \mathrm{i}=1,2,3 \text { y } 4
\end{gathered}
$$

$$
\delta\left(P_{1}, P_{i}\right)=\frac{1}{n} D\left(P_{1}, P_{i}\right) \text { distancia relativa de Hamming }
$$

\begin{tabular}{|c|c|c|}
\hline \multicolumn{3}{|c|}{ Cuadro 2. Distancias de Hamming } \\
\hline $\begin{array}{lrl}\text { Percepción de } & \text { AMLO } \\
\text { respecto al PRI } & \end{array}$ & $\begin{array}{l}\text { Distancia de } \\
\text { Hamming }\end{array}$ & $\begin{array}{l}D\left(P_{1}, P_{1}\right) \\
=3.200\end{array}$ \\
\hline & $\begin{array}{l}\text { Distancia relativa } \\
\text { de Hamming }\end{array}$ & $\begin{array}{c}\delta\left(P_{1}, P_{1}\right)= \\
0.200\end{array}$ \\
\hline $\begin{array}{lr}\text { Percepción de } & \text { AMLO } \\
\text { respecto al PAN } & \\
\end{array}$ & $\begin{array}{l}\text { Distancia de } \\
\text { Hamming }\end{array}$ & $\begin{array}{l}\mathrm{D}\left(\mathrm{P}_{1}, \mathrm{P}_{2}\right) \\
=3.600\end{array}$ \\
\hline & $\begin{array}{l}\text { Distancia relativa } \\
\text { de Hamming }\end{array}$ & $\begin{array}{c}\delta\left(P_{1}, P_{2}\right)= \\
0.225\end{array}$ \\
\hline $\begin{array}{lr}\text { Percepción de } & \text { AMLO } \\
\text { respecto al PRD } & \\
\end{array}$ & $\begin{array}{l}\text { Distancia de } \\
\text { Hamming }\end{array}$ & $\begin{array}{l}D\left(P_{1}, P_{3}\right) \\
=4.400\end{array}$ \\
\hline
\end{tabular}

Los resultados de dichos cálculos se muestran en el Cuadro 2:

\begin{tabular}{|l|l|c|}
\hline & $\begin{array}{l}\text { Distancia relativa } \\
\text { de Hamming }\end{array}$ & $\begin{array}{c}\delta\left(\mathrm{P}_{1}, \mathrm{P}_{3}\right)= \\
0.275\end{array}$ \\
\hline $\begin{array}{l}\text { Percepción de AMLO } \\
\text { respecto a MORENA }\end{array}$ & Distancia de & $\mathrm{D}\left(\mathrm{P}_{1}, \mathrm{P}_{4}\right)$ \\
& Hamming & $=0.900$ \\
\hline & Distancia relativa & $\delta\left(\mathrm{P}_{1}, \mathrm{P}_{4}\right)=$ \\
& de Hamming & 0.056 \\
\hline
\end{tabular}

Como se puede observar, la distancia relativa de Hamming entre los simpatizantes de MORENA y la imagen de un presidente ideal es de tan sólo 0.056 muy por debajo de cualquier distancia con los otros 3 partidos políticos. Recordemos que entre más pequeña es la distancia más cerca están lo subconjuntos borrosos.

De hecho, si deseamos poner de manifiesto un orden de acercamiento en la percepción de López Obrador en cada uno de los partidos políticos con respecto a la imagen del perfil ideal de un presidente, el orden se establece desde el partido con la distancia más pequeña, hasta el partido con la distancia más grande y quedaría así:

$\mathrm{P}_{1}($ presidente ideal $) \rightarrow \mathrm{P}_{4}(0.056-\mathrm{MORENA}) \rightarrow \mathrm{P}_{1}(0.200$ $\mathrm{PRI}) \rightarrow \mathrm{P}_{2}(0.225-\mathrm{PAN}) \rightarrow \mathrm{P}_{3}(0.275-\mathrm{PRD})$

\section{CONCLUSIONES: Consideraciones de Interés}

Con la distancia de Hamming pudimos comprobar la hipótesis que nos planteamos al inicio del documento: "Los simpatizantes del partido MORENA perciben a Andrés Manuel López Obrador como la figura más cercana al perfil de un presidente ideal, comparado con la percepción que tienen los simpatizantes de cualquier otro partido político".

De hecho, también se estableció un orden de acercamiento (o alejamiento), siendo el PRD el partido que percibe a López Obrador como la figura más lejana a un presidente ideal. Lo anterior puede explicarse por el hecho de que López Obrador abandonó las filas del PRD entre 2011 y 2012 para formar su propio partido MORENA; recordemos que en las elecciones del 2012, López Obrador realizó el segundo intento por ganar la presidencia de la república bajo la bandera del PRD. Ambos partidos políticos (PRD y MORENA) al ser de izquierdas, esto ha representado una crisis en el pensamiento político, que muchos simpatizantes continúan posiblemente sin sanar. 
Mauricio Ortigosa Hernández

Cabe aclarar que, si partimos de otras premisas, tales como: castigar si no se alcanza el nivel requerido, pero no premiar ni castigar si es rebasado el nivel requerido, o bien si cambiamos la distancia de Hamming, por otro criterio diferente de distancias, esto podría llevarnos a un orden distinto, aun cuando pudiéramos conservar a MORENA como el más cercano al perfil del presidente ideal.

Todo lo presentado en el presente documento, no es más que una puerta entreabierta para otros temas políticos que podrán modelarse bajo la óptica de la teoría de los subconjuntos borrosos.

\section{REFERENCIAS:}

Berrocal, S. (2004). Una aproximación a la nueva retórica del líder político televisivo: acciones, cualidades y discurso. Doxa Comunicación 2, 53-67.

D'Adamo, O., García Beaudoux, V. y Pastore, M. (2015 Marzo). EL "PRESIDENTE IDEAL" PARA LOS PORTEÑOS. Centro de Opinión Pública.

Deusdad, B. (2003). El concepto de liderazgo político carismático: Populismo e identidades. Opción, 19(41), 9-35. Recuperado en 18 de febrero de 2019, de http://www.scielo.org.ve/scielo.php?script=sci_arttext\&p id=S1012-15872003000200002\&lng=es\&tlng=es.

Diemidova, A., Adamovska, V. y Garasiuk, O. (2018). Effective Image of Modern Politician in the Context of Public Management and Administration. Scientific Periodicals. 2, 41-49.

García, V., D’Adamo, O. y Zubieta, E. (2016). Presidente ideal y Comunicación Política. Expectativas de los ciudadanos e imágenes proyectadas en los spots de las campañas presidenciales argentinas 2003, 2007 y 2011. Universidad Santiago de Compostela MARCO, 2, 1-23. http://dx.doi.org/10.15304/marco.2.2842

Gil Aluja, J. (2002). Introducción de la Teoría de la incertidumbre en la gestión de empresas. Vigo España: Editorial Milladoiro.
Kaufmann, A.; Gil Aluja, J. (1992). Técnicas de gestión de empresa: Previsiones, Decisiones y Estrategias. Madrid España: Ediciones Pirámide.

Kinder, D.R., Peters, M.D., Abelson, R.P. y Fiske, S.T. (1980). PRESIDENTIAL PROTOTYPES. Political Beahavior, 2(4), 315-337.

Martín Salgado, L. (2002). Marketing Político. Arte y ciencia de la persuasión en democracia. Barcelona España: Paidós Ibérica.

Merolla, J. y Zechmeister, E. (2009). Las percepciones de liderazgo en el contexto de las elecciones mexicanas de 2006. Política y Gobierno, Volumen Temático 1, 4181.

Ogneva Yu.l. y Kalmykov N.N. (2017). Expert Opinion: Image of the Ideal Politician in Russia. - Polis. Political Studies. 1 116-124. (In Russ.).DOl: https://doi.org/10.17976/jpps/2017.01.10

Orejuela S.S. (2009). Personalización política: la imagen del político como estrategia electoral. Revista de Comunicación, 8, 60-83.

Roets, A., y Van Hiel, A. (2009). The ideal politician: Impact of voters' ideology. Personality and Individual Differences, $\quad 46(1), \quad 60-65$. doi:10.1016/j.paid.2008.09.006

Zakrizevska, M. (2012). The social representations of the inhabitants of Latvia about the ideal image of a politician, EDP Sciences SHS Web of Conferences 2, 00042 DOI: 10.1051/shsconf/20120200042

Zamora, R. y Losada, J. (2011). La imagen del candidato electoral: Hacia una integración de su dimensión racional y emocional. Cuadernos de Información, 29, 924. 